\title{
In silico predictions of protein interactions between Zika virus and human host
}

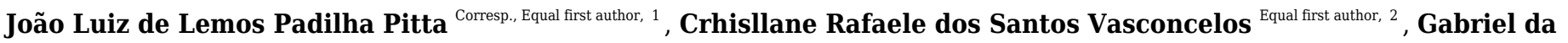 \\ Luz Wallau $^{3}$, Túlio de Lima Campos ${ }^{4}$, Antonio Mauro Rezende ${ }^{\text {Corresp. } 1}$ \\ 1 Microbiology Department, Aggeu Magalhães Institute - FIOCRUZ/PE, Recife, PE, Brasil \\ 2 Genetics Department, Federal University of Pernambuco, Recife, PE, Brasil \\ 3 Entomology Department, Aggeu Magalhães Institute - FIOCRUZ/PE, Recife, PE, Brasil \\ 4 Bioinformatics Platform, Aggeu Magalhães Institute - FIOCRUZ/PE, Recife, PE, Brasil \\ Corresponding Authors: João Luiz de Lemos Padilha Pitta, Antonio Mauro Rezende \\ Email address: joao.pitta@tjpe.jus.br, antonio.rezende@cpqam.fiocruz.br
}

Background. The ZIKA virus (ZIKV) belongs to the Flaviviridae family and was first isolated in the 40's, remained underreported until its global threat in 2016, where drastic consequences were reported as Guillan-Barre syndrome and microcephaly in newborns. Understanding molecular interactions of ZIKV proteins during host's infection is important to develop treatments and prophylactic measures. Largescale experimental approaches normally used to detect protein-protein interaction (PPI) are onerous and labor intensive. On the other hands, computational methods may overcome these challenges and guide traditional approaches on one or few protein molecules. The prediction of PPIs can be used to study the host-parasite interactions at the protein level and reveal key pathways that allows viral infection.

Results. Applying Random Forest and Support Vector Machine (SVM) algorithms, we performed predictions of PPI between two ZIKV strains and human proteomes. The consensus number of predictions of both algorithms was 17,223 pairs of proteins. Functional enrichment analyses were executed with the predicted networks in order to access the biological meanings on the protein interactions. Some pathways related to viral infection and neurological development were found for both ZIKV strains on the enrichment analysis, but JAK-STAT pathway was observed only for strain PE243 when compared with FSS13025 strain.

Conclusions. The consensus network of PPI predictions made by Random Forest and SVM algorithms allowed an enrichment analysis that corroborates with many aspects of ZIKV infection. The enrichment results are mainly related with viral infection, neuronal development and immune response and presented differences among the two ZIKV strains compared. Strain PE243 presented more predicted interactions between proteins from JAK-STAT signaling pathway, which could lead to a more intensive immune response when compared with strain FSS13025. These results show that the methodology employed here can reveals potentially new interactions between the ZIKV and humans' cells. 


\section{In silico predictions of protein interactions between} 3 Zika virus and human host

Corresponding Author:

João Luiz de Lemos Padilha Pitta ${ }^{1}$, Antonio Mauro Rezende ${ }^{1}$

Av. Professor Moraes Rego, s/n, Recife, Pernambuco, 50740-465, Brazil

Email address: jlpitta82@gmail.com, antonio.rezende@cpqam.fiocruz.br

\section{Abstract}

Background. The ZIKA virus (ZIKV) belongs to the Flaviviridae family, was first isolated in the 1940s, and remained underreported until its global threat in 2016, where drastic consequences were reported as Guillan-Barre syndrome and microcephaly in newborns. Understanding molecular interactions of ZIKV proteins during the host infection is important to develop treatments and prophylactic measures however, large-scale experimental approaches normally used to detect protein-protein interaction (PPI) are onerous and labor-intensive. On the other hand, computational methods may overcome these challenges and guide traditional approaches on one or few protein molecules. The prediction of PPIs can be used to study hostparasite interactions at the protein level and reveal key pathways that allow viral infection.

Results. Applying Random Forest and Support Vector Machine (SVM) algorithms, we performed predictions of PPI between two ZIKV strains and human proteomes. The consensus number of predictions of both algorithms was 17,223 pairs of proteins. Functional enrichment analyses were executed with the predicted networks to access the biological meanings of the protein interactions. Some pathways related to viral infection and neurological development were found for both ZIKV strains in the enrichment analysis, but the JAK-STAT pathway was observed only for strain PE243 when compared with the FSS13025 strain. Conclusions. The consensus network of PPI predictions made by Random Forest and SVM algorithms allowed an enrichment analysis that corroborates many aspects of ZIKV infection. The enrichment results are mainly related to viral infection, neuronal development, and immune response, and presented differences among the two compared ZIKV strains. Strain PE243 
40 presented more predicted interactions between proteins from the JAK-STAT signaling pathway, 41 which could lead to a more inflammatory immune response when compared with the FSS13025

42 strain. These results show that the methodology employed in this study can potentially reveal 43 new interactions between the ZIKV and human cells.

44 Keywords: zika virus; protein-protein interactions; machine learning; metabolic pathway 45 Introduction

46 Currently, there are approximately 80 species of arbovirus (arthropod-borne virus) that can infect humans. Arboviruses are a public health problem, mainly in tropical and developing countries, as seen by the annual epidemics of Dengue virus (DENV) as well as emergent arboviruses such as the Zika (ZIKV) and Chikungunya (CHIKV) viruses (Bichaud et al., 2014). ZIKV was highlighted in 2015 due to the high number of infections in Brazil and the correlation of ZIKV infection with increasing cases of Guillain-Barré syndrome and microcephaly in newborns (Ayres, 2016). Those facts led the World Health Organization to declare ZIKV a global health problem (WHO, 2016). Brazil had more than 200,000 reported cases of ZIKV at the peak of the 2016 emergency (Spitz, 2019). The number of cases has now decreased in Brazil, but ZIKV continues to circulate and there is still the possibility of further outbreaks. ZIKV is a single-stranded positive-sense RNA virus, and it belongs to the genus Flavivirus, with a genome of $10.794 \mathrm{bp}$ length encoding a polyprotein of approximately 3400 amino acids (Faye et al., 2014; Saiz et al., 2016; Sirohi et al., 2016). In its mature phase, the polyprotein is cleaved into three structural and seven non-structural proteins (Saiz et al., 2016; Sirohi et al., 2016). From a phylogenetic point of view, ZIKV has two major lineages: one includes the African strains and the other is the Asian strains (Saiz et al., 2016). Many studies have investigated and found the association between the virus infection and microcephaly (Hazin et al., 2016; Sirohi et al., 2016; Souza et al., 2016; de Araújo et al., 2017), and also the sexual transmission, transmission between domestic animals and humans, and different mosquito vectors involved with the virus transmission (Bueno et al., 2016; Aliota et al., 2017; Murray et al., 2017; Lowe et al., 2018). Despite all the knowledge acquired about ZIKV and the confirmation that the infection causes microcephaly (de Araújo et al., 2017), there are still gaps about the biological processes related to ZIKV infection and spreading throughout the human body. A better understanding of the molecular mechanisms behind these processes will certainly provide key information to elaborate or improve strategies to combat this pathogen. A way to increase the understanding of molecular events that take place between host and pathogens is the identification of protein interactions between them. However, the identification of protein interaction by experimental methods is a laborious process, costly, and subject to systematic errors, especially when applied on a large scale (Jansen \& Gerstein, 2004; Harrington, Jensen \& Bork, 2008). Due to these difficulties, computational methods emerge as important approaches once they are faster and cheaper. In addition, there is a daily increase in the amount

77 of molecular information available in public databases, providing the raw information needed to 78 generate more precise and sensitive computational models with greater capacity for 
80

81

82

83

84

85

86

87

88

89

90

91

92

93

94

95

96

97

98

99

100

101

102

103

104

105

106

107

108

109

110

111

112

113

114

115

116

117

generalization of methods and data representation. A sample of how fast new biological data has been deposited in different databases is presented by Goodacre et al. (Goodacre et al., 2020). Computational analysis for PPI has been used in the most different data sources in recent years, for instance, Shah et al. (Shah et al., 2018) and Scaturro et al. (Scaturro et al., 2018) used a more traditional affinity purification-mass spectrometry (AP-MS) approach to generate data and a computational methodology for build ZIKV PPI network. In Shah et al. (2018) flaviviruses were analyzed, including DENV and ZIKV, and conserved virus-host PPIs were compared to present evidence of the impacts on the immune system, while Scaturro et al. (2018) focused on the impacts of ZIKV infection on the neurological system. Other studies use a straight computational approach, collecting biological data from a public database and applying High-confidence Protein-Protein Interaction Prediction (HiPPIP) model (Ganapathiraju, Karunakaran \& CorreaMenéndez, 2016) or protein interaction prediction techniques based on domain interactions (Esteves et al., 2017). Ganapathiraju et al. (2016) analyzed the functional and pathway associations of the interacting proteins, trying to find potential targets of ZIKV for host invasion and Esteves et al. (2017) employed an algorithm to predict PPI and compare targets and strategies of ZIKV and other Flavivirus. There are still studies that apply a computational approach to analyze a broad number of viruses, including ZIKV, as proposed by Lasso et al. (Lasso et al., 2019).

Another useful set of approaches to predict PPI is the machine learning techniques. Lian et al. (Lian et al., 2019) employed the Random Forest technique to predict PPI between bacteria and host, while Ahmed et al. (Ahmed, Witbooi \& Christoffels, 2018) applied Support Vector Machine (SVM) to predict human-HPV PPI, and both used random sampling approach for building the negative dataset for the model.

Here, we used two machine learning methods (Random Forest and SVM) to predict protein interactions between two ZIKV strains and humans (Homo sapiens) applying the existing genomic and proteomic data for those organisms. A database of known validated protein interactions between different virus and hosts species was also used as a training set for the machine learning algorithms and the negative dataset used a dissimilarity-based negative sampling approach (Eid, ElHefnawi \& Heath, 2016) to build the negative dataset, avoiding noise caused by random sampling technique (Yang et al., 2020). The features we used to train machine learning algorithms, as Eid et al. (2016), were based on the amino acid physicochemical properties of the proteins involved in the interactions of the training dataset, and then the models were applied in the prediction of interactions between the proteins of ZIKV and human. The in silico approach used here makes it possible to test the whole human proteome against the ZIKV proteins, which would demand a much longer time and would be overly expensive if performed using experimental methods. This study aims to provide clues on how the pathogenesis caused by ZIKV happens through PPI analysis using a computationally efficient yet robust solution.

\section{Materials \& Methods}

\section{Biological data}


118 The Virus Pathogen database (https:/www.viprbrc.org/) (Pickett et al., 2012) was used to obtain 119 the ZIKV proteomes for the strains analyzed during the work. The strains PE243 (GenBank 120 KX197192.1) and strain FSS13025 (GenBank MH158236) are both ZIKV strains of Asian 121 lineages. The strain PE243 was isolated and sequenced in the state of Pernambuco - Brazil, 122 during the Brazilian outbreak of 2015, and it was associated with newborn microcephaly (Donald 123 et al., 2016). This strain has been used in many studies (Guedes et al., 2017; Pavithra L. Chavali

124

125

126

127

128

129

130

131

132

133

134

135

136

137

138

139

140

141

142

143

144

145

146

147

148

149

150

151

152

153

154

155

156

157 et al., 2017; Widman et al., 2017; Lima et al., 2019), and it was selected due to the local epidemiological importance. FSS13025 is a strain isolated from Cambodia in 2010 and it was selected due to the evolutionary distance when compared to PE243 (Widman et al., 2017), as it is not associated with microcephaly cases and has been used for comparison with PE243 in other studies (Aldo et al., 2016; Widman et al., 2017; Lima et al., 2019). In addition, several experimental tools and data on these two ZIKV strains were obtained in our previous study, including expression data for infected human cells (Lima et al., 2019). Besides the ZIKV, the proteome of Homo sapiens (ID UP000005640) without isoforms was downloaded from the UniProt database (https://www.uniprot.org/) (Bateman, 2019) on February 7, 2019.

\section{Training Interaction Dataset}

Classical machine learning approaches use positive and negative datasets to train classification algorithms. The present study uses a set of protein pairs interacting as a positive dataset. Those interactions come from the VirusMentha database (https://virusmentha.uniroma2.it/) (Calderone, Licata \& Cesareni, 2015), which describes interactions between proteins of viruses belonging to 25 viral families and proteins from 8 different hosts. All interactions present in that repository have been obtained experimentally, and VirusMentha was chosen due to the diversity of host proteins that include bacteria, plants, metazoan, and proteins of 25 families of viruses. The diversity of information present in VirusMentha is desired for the machine learning approaches applied here to address the generalization and avoid biases in the trained model. The interaction information downloaded from VirusMentha is a tabular file with a confidence score for each protein interaction, however, it does not contain amino acid sequences for the proteins. The sequences of each protein present in the interactions were downloaded from the UniProt database. Interaction and protein sequence data were downloaded on February 7, 2019.

Interactions in which one pair member was a viral polyprotein were removed due to the fact that viral polyproteins are cleaved to form viral mature proteins, and therefore have the amino acid sequence of all viral proteins, which could induce bias in the training data set. Interactions between two viral proteins were not used for the training dataset since the trained algorithms were used here to predict interactions between viral and host proteins. In addition, only interactions from VirusMentha with a confidence score higher than 0.3 were used, following the recommendations of Villaveces et al. 2015 (Villaveces et al., 2015).

The other step of dataset training is the negative dataset. The negative dataset is represented by pairs of proteins that probably do not interact. Selection of negative examples is a wellrecognized challenge for PPI prediction since biological datasets rarely include pairs of proteins that are known not to interact (Ben-Hur \& Noble, 2006; Dyer, Murali \& Sobral, 2011), and there 
158 is no gold standard to generate non-interactions set (Eid, ElHefnawi \& Heath, 2016; Ahmed,

159 Witbooi \& Christoffels, 2018; Yang et al., 2019). A common approach to generate non-

160 interactions sets is randomly chosen protein pairs that are not present in the set of interacting 161 proteins (Ben-Hur \& Noble, 2006; Eid, ElHefnawi \& Heath, 2016; Ahmed, Witbooi \& 162 Christoffels, 2018; Mei \& Zhang, 2019; Yang et al., 2019). However, previous studies have 163 shown that random pairing may cause noise, limiting the usability of such negative sample sets 164 (Eid, ElHefnawi \& Heath, 2016; Yang et al., 2020). Since random sampling produces more 165 incorrect negative examples than expected, degrading the learning process, and lowering the

166

167

168

169

170

171

172

173

174

175

176

177

178

179

180

181

182

183

184

185

186

187

188

189

190

191

192

193

194

195

196

197 prediction sensitivity, our goal was to implement a process that finds pairs of proteins most likely to be non-interactive for machine learning use. In this work, the negative dataset was derived from the data downloaded from VirusMentha following the rationale of Eid et al. (Eid, ElHefnawi \& Heath, 2016), however, some adjustments were applied. First, we applied a local alignment comparing all proteins versus all proteins present in the positive dataset using the Blastp program of the BLAST+ package version 2.2.30 (Camacho et al., 2009). Alignment parameters such as identity, similarity, and coverage were extracted, and only alignments with at least $80 \%$ of coverage between subject and query were used for the next steps. Clustering was also performed using CD-Hit version 4.6 (Fu et al., 2012) among the proteins from the positive dataset. A four-step approach was implemented for a viral protein "V" and host protein " $H$ " to be considered a pair of proteins that do not interact. In the first step, the pair of proteins "V-H" must not be listed as a pair present in the positive dataset. The second and third steps are based on the methodology presented at Eid et al. (Eid, ElHefnawi \& Heath, 2016), where the second step consists in verifying if the protein "V" does not interact with another host protein that has at least $20 \%$ identity with " $\mathrm{H}$ ". The third step verifies if the protein " $\mathrm{H}$ " does not interact with another viral protein that has at least $20 \%$ identity with "V". Additionally to what is proposed in Eid et al. (Eid, ElHefnawi \& Heath, 2016), a fourth step verifies if another pair of a viral protein "A" and host proteins "B", where "A" has at least $40 \%$ of identity with "V" and "B" has at least $40 \%$ of identity with " $\mathrm{H}$ ", is listed in the positive dataset. Therefore, if the pair "A-B" has at least $40 \%$ of identity with pair "V-H", the pair "V-H" is discarded alike a pair from the negative dataset due to the identity with pair "A-B" present in the positive dataset. Figure 1 shows the four steps approach.

\section{Figure 1. Four steps approach for negative pair of proteins.}

The four steps to set a pair of one viral protein "V" and one host's protein " $H$ " as proteins that do not interact. The first step (A) verifies if the pair of proteins is not listed as a pair on the positive interaction dataset. The second step (B) consists in to verify if the "V" protein does not interact with another host protein "Hx" that has more than $20 \%$ identity with "H". Third step (C) checks also if the protein " $\mathrm{H}$ " does not interact with another viral protein "Vx" that has more than $20 \%$ identity with "V". The fourth step (D) verifies if another pair, composed by a viral protein " $\mathrm{A}$ " that has at least $40 \%$ of identity with " $\mathrm{V}$ " and a host protein "B" that has at least $40 \%$ of identity with " $\mathrm{H}$ ", is listed as positive interaction in the positive dataset. The pair "V-H" is discarded 
198

199

200

201

202

203

204

205

206

207

208

209

210

211

212

213

214

215

216

217

218

219

220

221

222

223

224

225

226

227

228

229

230

231

232

233

234

235

236

237

from the negative dataset if it has at least $40 \%$ identity with pair "A-B" and "A-B" is listed in the positive dataset.

The thresholds of steps two and three were set in $20 \%$ of identity as below that, the structural similarity is minimal (Eid, ElHefnawi \& Heath, 2016). The $40 \%$ of identity used during the clusterization was set as it is the smaller threshold allowed by CD-HIT and the evaluation of identity between pairs aims to place in negative dataset pairs with the greatest possible difference of pairs listed in the positive dataset. The same number of positive pairs were generated following the steps above for the negative dataset, avoiding bias during algorithm training as proposed by Shen et al. (Shen et al., 2007).

\section{Algorithm training}

Protein interaction predictions were performed using two classical techniques of machine learning: Support Vector Machine (SVM) and Random Forest. SVM and Random Forest were selected since both are well-validated algorithms for binary classification and have been used in many studies for the classification of biological data (Statnikov \& Aliferis, 2007; Statnikov, Wang \& Aliferis, 2008; Gajowniczek et al., 2020). We used the Random Forest algorithm as prediction tools implemented in Caret package version 6.0-78 for R (Kuhn, 2013), and SVM classification was done using LIBSVM version 3.22 (Chih-Wei Hsu, Chih-Chung Chang, 2008) as the implementation of the SVM algorithm. The Random Forest algorithm was applied using two different modes named "class" and "prob". When mode "class" is used, the interaction prediction is given as label " 0 " for negative, or " 1 " for positive. Mode "prob" returns the probability of non-interaction associated with the prediction.

The features, based on physicochemical properties of proteins used to train both machine learning algorithms, were extracted using the R package protR version 1.4-2 (Xiao et al., 2015). Briefly, amino acid sequences of the proteins present in the training dataset were loaded into the $\mathrm{R}$ environment using the RStudio as IDE (Integrated Development Environment). The R package protR was first used to check if all the amino acid sequences had only the 20 valid amino acids and sequences that did not respect that were removed from further steps. Afterward, amino acids in the protein sequences were classified into 7 groups, based on the physicochemical characteristics (e.g. dipoles and side-chain volume), and each group received a label as proposed by Cui et al. (Cui, Fang \& Han, 2012). Table 1 shows the groups of amino acids and respective labels.

\section{Table 1. Classification of amino acids in group labels.}

The protein sequences of amino acids became sequences of group labels, following the categorization scheme present in Table 1. After that, each sequence was analyzed in a triad of labels, and the frequency of each triad is calculated as proposed by Shen et al. (2007) (Shen et al., 2007), applying a normalization of the frequencies in values between 0 and 1 . Thus, the sequences were converted into a vector of 343 positions (seven groups arranged in triads, so 
238

239

240

241

242

243

244

245

246

247

248

249

250

251

252

253

254

255

256

257

258

259

260

261

262

263

264

265

266

267

268

269

270

271

272

273

274

275

276

277

$7^{3}=343$ ). Therefore, the generated vectors had the same size allowing comparison between proteins with different sequence lengths. The output of protR with the normalized vectors was used as features for Random Forest and SVM algorithms training. The CSV (Comma-separated values) files with the vectors of features used to training both algorithms can be found in the Supplemental Information (see Supplemental Dataset S1 for the negative dataset in Random Forest, Supplemental Dataset S2 for the positive dataset in Random Forest, and Supplemental Dataset S3 for negative and positive datasets in SVM).

During the training steps, the dataset was divided into two parts, one with $75 \%$ of the dataset used as the training set, and the other with $25 \%$ was used as the test set for validation of the model. The proportions of positive and negative samples of interactions were kept for both parts. Training steps were performed using 5-fold cross-validation for both algorithms, shuffling the interactions present in both sets former cited. A radial kernel function was applied for the SVM algorithm, which required a definition of gamma and sigma values in the model construction. Thus, a grid search for parameter selection was performed using grip.py (Chih-Wei Hsu, ChihChung Chang, 2008) script of LIBSVM aiming to determine the best values of sigma and gamma based on the training dataset. The results of classification for the dataset used as test set during the 5-fold cross-validation was used to calculate the accuracy, sensitivity, and specificity of the model. The model built for both algorithms, including models for modes "class" and "prob" of Random Forest algorithm, is available in the Supplemental Information (see Supplemental Model S1 for SVM, Supplemental Model S2 for Random Forest mode "class" and Supplemental Model S3 for Random Forest mode "prob").

\section{Prediction of Protein Interactions}

The ZIKV strains and human (H. sapiens) proteins were processed following the same steps for proteins present in the training dataset. Briefly, the protein sequences were loaded and protR was applied again to verify if all proteins had only valid amino acids. Sequences were converted into vectors representing the physicochemical features. Those vectors were combined in a pair-wise manner being one member belonging to $H$. sapiens and the other member to one of the two ZIKV strains. The constructed models for Random Forest and SVM were used to classify 586,572 pairs of proteins, being 28 proteins from both ZIKV strains against all 20,949 proteins from the human proteome.

\section{Analysis of data enrichment}

A consensus prediction was constructed based on the predictions made by both algorithms, The consensus consisted of all positive predictions in Random Forest using mode "class", positive in SVM, and smaller than 0.5 in Random Forest using mode "prob". The human proteins present at the consensus predicted interactions for both ZIKV strains were submitted to perform functional enrichment analysis on the DAVID webservice version 6.8 (https://david.ncifcrf.gov/home.jsp) (Jiao et al., 2012). The enrichment analysis performed here is a technique to verify if the frequency of a KEGG (Kyoto Encyclopedia of Genes and Genomes (https://www.genome.jp/kegg) pathway describer term was higher than expected in the list of 
278 human proteins predicted to interact with ZIKV proteins, indicating a biological involvement of

279 such pathways in the ZIKV infection events. Therefore, the analysis was performed following 280 the rationale that a set of metabolic pathways could be affected by the interactions of proteins 281 with ZIKV proteins and this fact could provide evidence and clues on how the virus is behaving 282 inside the human host. The complete list of enriched pathways with their p-value and FDR (False 283 Discovery Rate), including non-statistically relevant terms for both strains, is available in the 284 Supplemental Information (Supplemental Table S1).

285 We opted to use KEGG (Ogata et al., 1999; Kanehisa, 2019; Kanehisa et al., 2019) terms to 286 describe the enrichment analysis results since KEGG is a knowledge base for systematic analysis 287 of gene functions, linking genomic information with higher-order functional information (Ogata 288 et al., 1999), and it is a reference for biological interpretation of genome sequences and other 289 high-throughput data (Kanehisa et al., 2019). Besides genomic and functional information, 290 KEGG provides terms that are straightforward for biological interpretation. Only terms with a $p$ 291 value smaller than 0.05 , adjusted by FDR of 5\%, were considered relevant for this study. In the 292 Supplemental Information is possible to find the set of pathway images from KEGG's database 293 with enriched statistically relevant data (see Supplemental Fig. S1 for strain PE243 and 294 Supplemental Fig. S2 for strain FSS130025).

\section{PPI network}

296 The consensus predictions were used to build a PPI network applying the Cytoscape 3.6 tool 297 (Shannon et al., 2003). The topology of the network was built in such a way that ZIKV proteins 298 of both strains analyzed were merged in the network. The human proteins were divided into 299 proteins that interact with only strain PE243, proteins that interact only with strain FSS13025, 300 and proteins that interact with both strains. The complete PPI network is available on 301 Supplemental Information (see Supplemental Fig S3).

\section{Results}

303 The file downloaded from VirusMentha had 11,776 interactions described. Once the

304

305

306

307

308

309

310

311

312

313

314

315

316

317 polyproteins and interactions between any two viral proteins were removed, a total of 4,157 interactions were kept. During the ProtR pattern checking step, a viral protein and seven host proteins were discarded, and 4,150 interactions were left. Since the interaction score of confidence was set as 0.3 , a total of 348 interactions were used as a positive set during algorithm training. The negative set was derived based on the VirusMentha database (11,776 interactions), and 1,040,816 negative interactions were produced using the methodology applied here. Aiming to keep the balance between the number of pairs in both positive and negative training sets, 348 pairs of negative interactions were randomly selected to be used in algorithm training. Merging positive and negative for the training of algorithms, a total of 696 pairs with a higher level of confidence in the interaction were obtained.

Based on 5-fold cross-validation approach, the specificity, sensitivity, and accuracy of the Random Forest approach were calculated being equal to $82.27 \%, 74.73 \%$, and $78.16 \%$, respectively. The grid search for parameter optimization to SVM obtained a gamma of 0.125 , sigma 2.0 , and an accuracy of $77,29 \%$. 
318 Taking the consensus prediction between the two Random Forest modes and SVM, a total of 319 17,223 pairs of proteins were predicted as positive interactions, being 7,932 for ZIKV strain 320 FSS13025 and 9,291 for PE243. Figure 2 shows the number of consensus predictions of ZIKV 321 strain and $H$. sapiens.

322

323 Figure 2. Number of interactions predicted.

324 The number of interactions predicted by the consensus prediction was analyzed and described distributing the interactions by strain. Red and blue rectangles are structural and nonstructural ZIKV proteins, respectively.

327

328

329

The complete table of PPI present on Fig. 2 is available on Supplemental Table S2. The interactions were grouped to visualize the number of human proteins that interact specifically

330

331

332

333

334

335

336

337

338

339

340

341

342

343

344

345

346

347

348

349

350

351

352

353

354

355

356

with one or both ZIKV strains as Fig. 3 shows. It is possible to see a greater number of specific interactions $(1,384)$ for the PE243 ZIKV strain. The main reason for that is the number of interactions for pr protein when both strains are compared.

\section{Figure 3. Numbers of interactions per strain.}

The Venn Diagram shows the interactions of human proteins per ZIKV strain. The model predicted 25 exclusive interactions for strain FSS13025 while 1,384 exclusive interactions were predicted for strain PE243. The model predicted yet 15,814 interactions for both strains.

The human proteins present in the final interaction predictions were used as input to functional enrichment analysis on DAVID Bioinformatics Resources 6.8 (https://david.ncifcrf.gov) (Jiao et al., 2012). The complete table with all the enrichment results, including non-statistically significant ones, can be accessed on Supplemental Table S1. The enrichment analysis returned 103 and 101 terms for FSS13025 and PE243 ZIKV strains, respectively. Pathways associated with neural development as PI3K-Akt signaling, Ras signaling, NF-kappa B signaling, and Axon guidance were found enriched during the analysis. Terms associated with viral infection and viruses related to TORCH syndrome such as Herpes simplex infection were also enriched. It was possible to recover terms associated with the ZIKV infection such as endocytosis and apoptosis process. Interestingly, PE243 presented a term related to the JAK-STAT signaling pathway process while FSS13025 did not. Figure 4 shows the enriched pathways with statistical relevance found and the different pathways for each strain.

\section{Figure 4. Enriched pathways with statistical relevance.}

Figure 4 presents a dot plot graphic for each strain. The size of each dot represents the number of genes ("Count") from the consensus prediction enriched in each pathway and the color presents the statistical relevance based on the FDR. 


\section{Discussion}

358 The present study applied an approach easy to deploy using classical machine learning

359

360

361

362

363

364

365

366

367

368

369

370

371

372

373

374

375

376

377

378

379

380

381

382

383

384

385

386

387

388

389

390

391

392

393

394

395

396

algorithms yet robust and fast for large-scale analysis. Aiming to build a better training set, interactions with the virus polyprotein and interactions between any two virus proteins were removed from the training set to avoid biases in the algorithm training phase, due to the fact that polyproteins have the sequence of all proteins and the present study is virus-host protein interaction classification. In addition, interactions with a confidence score value below the optimal value of 0.3 were discarded according to the cut-off point reported in Villaveces et al. (Villaveces et al., 2015), leaving a final set of 348 positive interactions.

Regarding the negative training set, we used the rationale proposed by Eid et al. (Eid, ElHefnawi \& Heath, 2016), instead of the common random sampling approach, to build a negative set with fewer false-negative samples as possible. This approach has been used in other studies like in Yang et al. (Yang et al., 2020) and it implements a more accurate heuristic approach to generate non-interactive pairs. The methodology based on the study of Eid et al. (Eid, ElHefnawi \& Heath, 2016) was applied, but unlike it, local alignment was used rather than a global strategy. The reason behind that is because specific protein domains may be important for the interactions (Singhal \& Resat, 2007). Moreover, an additional step was implemented, comparing our approach to the one of Eid et al. (Eid, ElHefnawi \& Heath, 2016), to improve the confidence on negative interaction pairs. Therefore, to increase reliability, the sequence identity of possible pairs in the negative dataset was compared with pairs from the positive dataset to avoid not only identical but also similar positive interaction pairs. In the end, we had 1,040,816 negative pairs as a set of negative samples, and we selected 348 for the training of the algorithm to maintain the generalization of the model. The number of samples from the negative set was set equal to that of the positive set to avoid bias in the construction of the model as proposed in Shen et al. (Shen et al., 2007).

The process of extraction of physicochemical characteristics and normalization is similar to that used in other studies (Dyer, Murali \& Sobral, 2007; Cui, Fang \& Han, 2012), using the physicochemical characteristics extraction model proposed in Shen et al. (Shen et al., 2007). This model is the state of the art for this purpose in the type of data used here. Proteins with nonstandard amino acids were discarded, and the others were properly normalized using the Rimplementation described in Xiao et al. (Xiao et al., 2015). The proteomes of two ZIKV strains were compared to the $H$. sapiens proteome, but since physicochemical information of proteins is the core information, this approach could be used with any ZIKV strain, any virus, or any organism proteome that makes sense in biological terms.

The accuracy of the model was compatible with that of the methodology used in Eid et al. (Eid, ElHefnawi \& Heath, 2016), when using $75 \%$ of the interaction data in the training set and 5 -fold cross-validation, and consequently comparable with other works for prediction between virus and host or prediction intraspecies. The results also showed that the increase in the number of cross-validation times for the dataset analyzed in this study did not improve significantly the accuracy of the model, contrary to what happens in other studies such as the one that applied

Peer) reviewing PDF | (2020:11:55456:3:0:NEW 20 Jun 2021) 
397

398

399

400

401

402

403

404

405

406

407

408

409

410

411

412

413

414

415

416

417

418

419

420

421

422

423

424

425

426

427

428

429

430

431

432

433

434

435

436

cross-validation in the maximum likelihood of crystallographic simulated annealing refinement data (Adams et al., 1997).

The results regarding the number of interactions predicted by the model as positive are comparable to other studies that use as methodology Bayesian inference and protein interaction prediction techniques based on domain interactions (Esteves et al., 2017). Yoon et al. (Yoon et al., 2017) found 143 protein interactions between ZIKV and H. sapiens in vitro. Since other studies, including the present one, predict thousands of interactions in silico, there are strong indications that there is still much to be discovered about the molecular processes of infection by the virus.

Analyzing the number of interactions predicted by the model per ZIKV protein, it was possible to observe that the envelope protein of the strain FSS13025 was predicted with only one interaction and no interaction with the strain PE243. It is known that the envelope protein interacts with human proteins, thus we further investigated the prediction data. We observed a positive prediction between an envelope protein and protein ID P08709 (Coagulation factor VII) for the strain FSS13025. On the other hand, the strain PE243 got a score of 0.5 using Random Forest "prob" mode, which is exactly the threshold value for the same protein ID P08709. In addition, that interaction had a negative prediction for Random Forest "class" mode and a positive prediction for the SVM tool. Those facts show how difficult it is to predict interactions at the borderline score scale of the prediction tools. Therefore, it should be considered the fact that the model has an accuracy of around $78 \%$ when the two algorithms are merged in the consensus prediction. In parallel, that prediction found might be important since coagulation disorders in ZIKV infection have been reported as responsible for abnormal fetal growth due to reduced perfusion of the umbilical cord (Anfasa et al., 2019), and delayed fetal brain maturation (Scher, 2019).

Observing the Fig. 3 is possible to notice that strain PE243 showed a much higher number of exclusive interactions when compared with the exclusive interactions for the FSS13025 strain, which might corroborate that the PE243 strain is more virulent and associated with disorders like microcephaly when compared with the strain FSS13025 (Lima et al., 2019). A larger number of PE243's exclusive interactions happen with one ZIKV protein and, observing Fig. 2, pr protein is the one that has this larger number of interactions in strain PE243 when compared with FSS13025. Protein prM is associated with ZIKV virulence (Nambala \& Su, 2018) and is described for flaviviruses that after cleavage of prM in pr and $\mathrm{M}$ proteins, pr remains attached to the virion until exocytosis, preventing premature fusion in the trans-Golgi network ( $\mathrm{Yu}$ et al., 2009; Zheng, Umashankar \& Kielian, 2010), therefore helping the exportation of fully functional viral particles. Another interesting observation about the number of interactions of pr protein is that $\mathrm{pr}$ is a small protein when compared with others ZIKV proteins, and it was expected that a bigger protein, and consequently with a larger surface, would have more interactions. Size, however, is not the sole determinant and there are other examples in the literature describing, for instance, that hemoglobin exhibits greater surface activity than the much larger fibrinogen protein (Dee, Puleo \& Bizios, 2002). 
437 Since metabolic pathways can be affected by the interactions between ZIKV and human proteins, 438 enrichment analysis, using the list of proteins ID of human proteome predicted as interacting 439 with ZIKV proteins, was performed aiming to provide evidence and clues on how the virus is 440 behaving inside the human host. Several pathways were identified as being affected by the 441 interaction of ZIKV and human proteome. Some of them were found just for one out of two 442 strains used in this work and others were recovered for both strains. Regarding the pathways 443 found for both viruses, we noticed the presence of important pathways already described as 444 being involved in virus infection such as the Inositol phosphate metabolism. This pathway has 445 already been described as an important pathway for rabies virus (RABV), which causes acute 446 encephalitis. Besson, B. et al. (Besson et al., 2019) showed phosphatidylinositol metabolism as the prominent factor for RABV infection, and those findings were confirmed in human neurons.

451

452

453

454

455

456

457

458

459

460

461

462

463

464

465

466

467

468

469

470

471

472

473

474

475 Another interesting pathway that had several proteins involved (enriched) is the Fanconi anemia. More than half of proteins that participate in this pathway were predicted to interact with ZIKV proteins. This pathway is related to microcephaly and mental retard (Krakow, 2013). The ErbB signaling pathway is a clear enriched pathway for ZIKV since several viruses were described using this family of receptors to get into the cell. In addition, some viruses also can use this pathway to alter the cell cycle changing the speed at which they multiply (Ho et al., 2017). Moreover, we could see proteins from this pathway related to Glioma differentiation interacting with ZIKV proteins as shown in Fig. 5.

\section{Figure 5. ErbB signaling pathway map.}

Figure 5 shows ErbB signaling pathway map. Enriched proteins in our data were marked in green over the pathway map. Green boxes represent proteins that were enriched by DAVID analysis between ZIKV (PE243 strain) and H. sapiens interactions.

Finally, this pathway is involved in the activation of one of the main intracellular signaling pathways of the human metabolome such as the NF-kappa B signaling pathway, PI3K-Akt signaling pathway, and Phosphatidylinositol signaling system, all of them being found enriched for our data. Interestingly, another central pathway called the Ras signaling pathway was also enriched. It has already been described as an important pathway for some viruses such as Herpesvirus, and it is involved with several important biological processes such as differentiation, proliferation, and cell survival (Filippakis, Spandidos \& Sourvinos, 2010). In our prediction, most proteins present in this pathway were predicted interacting with ZIKV proteins. We could see some expected enriched pathways normally modulated in viral infections such as apoptosis, cell cycle, and endocytosis, however, some more peculiar pathways were also identified such as the axon guidance pathway. The main cell controlling the axonal guidance is the astrocyte, and it is one of the targets of ZIKV infection (Limonta et al., 2018; Sher, Glover \& Coombs, 2019). This could be involved directly in microcephaly symptoms, and we could see several proteins from the host interacting with ZIKV proteins (Limonta et al., 2018). 
476 Another interesting pathway that deserves a highlight is the complement and coagulation 477 cascades. Some symptoms such as the rash on the skin (macules and papules) can be related to 478 the interference in this pathway. It is worth mentioning ZIKV and Dengue virus belong to the 479 same family, and those pathways are very disturbed on Dengue virus infection as it causes

480

481

482

483

484

485

486

487

488

489

490

491

492

493

494

495

496

497

498

499

500

501

502

503

504

505

506

507

508

509

510

511

512

513

514

515 dengue hemorrhagic fever (Rigau-Pérez et al., 1998). The extracellular matrix (ECM) interaction pathway along with focal adhesion structures are important sets of ZIKV protein interactions once the contact among cells (infected and uninfected) is important to viral spreading (Mothes et al., 2010). Viruses such as measles also can cause an acute inflammatory process in neurons, the contact between cells is important to guarantee the virus spreading (Lawrence et al., 2000). Moreover, it is possible to notice using the KEGG representation of ECM and focal adhesion pathway how they are connected and how pathways already mentioned here, which are disturbed by ZIKV infection, are influenced. One example is the phosphatidylinositol signaling system, showing how the virus infection has a considerable impact on normal cell functioning. Another impacted pathway in which many proteins are interacting with ZIKV proteins is ubiquitin-mediated proteolysis. Several viruses are known to manipulate the proteasome pathways aiming to change the cell cycle, inhibit apoptosis, evade the immune system, and activate cell signaling, helping the persistence of viral infection and viral carcinogenesis (Shoji, 2012).

Some cancer pathways were highlighted by enrichment analysis. One possibility is because all cancer pathways which appeared here, include other pathways already mentioned. This is clear in the KEGG pathway called "Pathways in Cancer" where all pathways represented in this scheme are interacting with proteins of ZIKV.

It is known that viral infection can affect insulin signaling pathways as it is modulated by the production of interferon- $\gamma$, a common molecule present in viral infection. However, the ZIKV was able to induce an insulin resistance pathway which can be a pathway activated as an immune response process (Šestan et al., 2018). In addition, this can be related to pancreatic cancer pathways, as the virus seems to induce an increase in insulin production.

On the other side, we had few pathways that were exclusively enriched for one of the strains of ZIKV studied here. The main one is the JAK-STAT signaling pathway. The JAK-STAT transducers and activators of transcription (JAK/STAT) pathway is one of a handful of pleiotropic cascades used to transduce a multitude of signals for development and homeostasis in animals, from humans to flies, and is considered the principal signaling mechanism for a wide array of cytokines and growth factors in mammals (Rawlings, Rosler \& Harrison, 2004). Once the cognate receptor of STAT is bound to cytokines, it is activated by members of the JAK family of tyrosine kinases and, after activated, they dimerize and translocate to the nucleus and modulate the expression of target genes (Kisseleva et al., 2002). In addition to the activation of STATs, JAKs mediate the recruitment of other molecules such as the MAP kinases, PI3 kinase, etc. These molecules process downstream signals via the Ras-Raf-MAP kinase and PI3 kinase pathways which results in the activation of additional transcription factors (Kisseleva et al., 2002). We could see more proteins from the JAK-STAT signaling pathway interacting with 
516 proteins of ZIKV strain PE243 compared to the protein of strain the FSS13025, which could lead 517 to a more disturbed immune response in the first one than in the second one. Interferons (IFNs)

518 are the main group of proteins responsible for an innate immune response during viral infection 519 and JAK/STAT is the main pathway activated by the IFNs (Nan, Wu \& Zhang, 2017). A 520 previous work of our group (Lima et al., 2019) shows that the PE243 strain can induce a higher 521 expression of IFN- $\alpha$, probably modulating the response of the JAK/STAT pathway stronger than 522 the modulation performed by the FSS13024 strain.

523 At the end, it is worth mentioning our results are corroborated at some level by other works with

524

525

526

527

528

529

530

531

532

533

534

535

536

537

538

539

540

541

542

543

544

545

546

547

548

549

550

551

552

553

554

555 different methodologies, including a previous work of our group (Lima et al., 2019). A clear example appears in Scaturro et al. (Scaturro et al., 2018), since they found the modulation of proteins such as protein kinase B, also known as AKT, and ATM-ATR complex is regulated by ZIKV infection, and those proteins are also present in our results as interacting with ZIKV proteins. Another example is found in Shah et al. (Shah et al., 2018) where pathways such as regulation of apoptosis and ubiquitin-mediate protein degradation were found just like in our results. Scaturro et al. (Scaturro et al., 2018) and Shah et al. (Shah et al., 2018) used a more traditional approach with affinity purification coupled with liquid chromatography and tandem mass spectrometry to identify interactions between human and ZIKV proteins. Despite this methodological difference compared to our in silico approach, we could observe some overlaps about the AKT-mTOR signaling pathway, cell-cycle regulation pathway, and more specifically the modulation of ATM/ATR proteins in this pathway associated with the DNA-damage pathway. Other overlaps can be found when compared to our study with others that use the methodology in silico. Ganapathiraju et al. (Ganapathiraju, Karunakaran \& Correa-Menéndez, 2016) found pathways such as toll-like receptor signaling, axonal guidance signaling, and actin cytoskeleton signaling, all found in our results, while Esteves et al. (Esteves et al., 2017) found numbers of PPI predictions comparable with our numbers. Even in Lasso et al. (Lasso et al., 2019), where many viruses including ZIKV were evaluated, apoptosis, cell-cycle, JAK-STAT, and NF- $\kappa \mathrm{B}$ pathways were "recurrently targeted across all viruses" and overlaps with our results. Those facts can indicate the robustness of the approach used here to predict protein interactions.

\section{Conclusions}

Since the success of the machine learning approach depends on the set used to train the model, the present study deployed, with some improvements, a methodology described in the literature with a positive sample set based on experimental data. The negative samples were derived from the positive data using an approach focusing on the probability that a pair is non-interactive. The consensus network of PPI predictions made by Random Forest and SVM algorithms allowed an enrichment analysis that corroborates many aspects found in literature about ZIKV infection. The pathways presented on enrichment analysis are mainly related to viral infection, neuronal development, and immune response, all compatible with ZIKV infection, and there were different pathways among the two ZIKV strains compared. The strain PE243 showed more predicted interactions between proteins from the JAK-STAT signaling pathway when compared with the strain FSS13025, being this pathway specifically enriched for strain PE243, which could 
556 lead to a more disturbed immune response to this strain. In addition, some pathways were

557 supported by experimental evidence in previous work of our group. These results show that the

558 methodology employed here is solid, can reveal potential new interactions between the ZIKV

559 and human cells, and can be used in other viral-host protein interaction studies.

560 Acknowledgments

561 The authors thank FIOCRUZ-PE for the use of its facilities and the Bioinformatics research

562 group for all the support.

563 Funding

564 This work was supported by Fundação Oswaldo Cruz (FIOCRUZ). The computational platform

565 used for apply the methodology described in this study was funded by Coordenação de

566 Aperfeiçoamento de Pessoal de Nível Superior (CAPES, Brazil).

\section{Author Contributions}

568

569

570

571

572

573

574

575

576

577

578

579

580

581

582

583

584

585

586

587

588

589

590

591

592

593

594

595

596

- João Luiz de Lemos Padilha Pitta - Data curation, formal analysis, methodology, validation, writing - original draft

- Crhisllane Rafaele dos Santos Vasconcelos - Formal analysis, methodology, writing review \& editing

- Gabriel da Luz Wallau - Methodology, writing - review \& editing

- Túlio de Lima Campos - Methodology, writing - review \& editing

- Antonio Mauro Rezende - Conceptualization, formal analysis, methodology, Project administration, Resources, Supervision, writing - original draft, writing - review \& editing

\section{Competing interests}

The authors declare that they have no competing interests.

\section{Supplemental Informations}

Supplemental information for this article can be found in supplemental files.

\section{References}

Adams PD, Pannu NS, Read RJ, Brünger AT. 1997. Cross-validated maximum likelihood enhances crystallographic simulated annealing refinement. DOI: 10.1073/pnas.94.10.5018.

Ahmed I, Witbooi P, Christoffels A. 2018. Prediction of human-Bacillus anthracis proteinprotein interactions using multi-layer neural network. Bioinformatics (Oxford, England) 34:4159-4164. DOI: 10.1093/bioinformatics/bty504.

Aldo P, You Y, Szigeti K, Horvath TL, Lindenbach B, Mor G. 2016. HSV-2 enhances ZIKV infection of the placenta and induces apoptosis in first-trimester trophoblast cells. American Journal of Reproductive Immunology 76:348-357. DOI: 10.1111/aji.12578.

Aliota MT, Bassit L, Bradrick SS, Cox B, Garcia-Blanco MA, Gavegnano C, Friedrich TC, Golos TG, Griffin DE, Haddow AD, Kallas EG, Kitron U, Lecuit M, Magnani DM, Marrs C, Mercer N, McSweegan E, Ng LFP, O'Connor DH, Osorio JE, Ribeiro GS, Ricciardi M, Rossi SL, Saade G, Schinazi RF, Schott-Lerner GO, Shan C, Shi PY, Watkins DI, Vasilakis N, Weaver SC. 2017. Zika in the Americas, year 2: What have we learned? What gaps remain? A report from the Global Virus Network. Antiviral Research 144:223-246. DOI: 10.1016/j.antiviral.2017.06.001.

Peer] reviewing PDF | (2020:11:55456:3:0:NEW 20 Jun 2021) 
597

598

599

600

601

602

603

604

605

606

607

608

609

610

611

612

613

614

615

616

617

618

619

620

621

622

623

624

625

626

627

628

629

630

631

632

633

634

635

636

637

638

639

640

641

642

Anfasa F, Goeijenbier M, Widagdo W, Siegers JY, Mumtaz N, Okba N, Van Riel D, Rockx B, Koopmans MPG, Meijers JCM, Martina BEE. 2019. Zika virus infection induces elevation of tissue factor production and apoptosis on human umbilical vein endothelial cells. Frontiers in Microbiology 10:1-11. DOI: 10.3389/fmicb.2019.00817.

de Araújo TVB, Ximenes RA de A, Miranda-Filho D de B, Souza WV, Montarroyos UR, de Melo APL, Valongueiro S, de Albuquerque M de FPM, Braga C, Filho SPB, Cordeiro MT, Vazquez E, Cruz D di CS, Henriques CMP, Bezerra LCA, Castanha PM da S, Dhalia R, Marques-Júnior ETA, Martelli CMT, Rodrigues LC. 2017. Association between microcephaly, Zika virus infection, and other risk factors in Brazil: Final report of a casecontrol study. The Lancet Infectious Diseases. DOI: 10.1016/S1473-3099(17)30727-2.

Ayres CFJ. 2016. Identification of Zika virus vectors and implications for control. The Lancet Infectious Diseases 16:278-9. DOI: 10.1016/S1473-3099(16)00073-6.

Bateman A. 2019. UniProt: A worldwide hub of protein knowledge. Nucleic Acids Research 47:D506-D515. DOI: 10.1093/nar/gky1049.

Ben-Hur A, Noble WS. 2006. Choosing negative examples for the prediction of protein-protein interactions. BMC Bioinformatics 7:1-6. DOI: 10.1186/1471-2105-7-S1-S2.

Besson B, Kim S, Kim T, Ko Y, Lee S, Larrous F, Song J, Shum D, Grailhe R, Bourhy H. 2019. Kinome-Wide RNA Interference Screening Identifies Mitogen-Activated Protein Kinases and Phosphatidylinositol Metabolism as Key Factors for Rabies Virus Infection. DOI: $10.1128 / \mathrm{mSphere.}$

Bichaud L, de Lamballerie X, Alkan C, Izri A, Gould EA, Charrel RN. 2014. Arthropods as a source of new RNA viruses. Microbial Pathogenesis 77:136-141. DOI: 10.1016/j.micpath.2014.09.002.

Bueno MG, Martinez N, Abdalla L, Duarte dos Santos CN, Chame M. 2016. Animals in the Zika Virus Life Cycle: What to Expect from Megadiverse Latin American Countries. PLoS Neglected Tropical Diseases 10. DOI: 10.1371/journal.pntd.0005073.

Calderone A, Licata L, Cesareni G. 2015. VirusMentha: A new resource for virus-host protein interactions. Nucleic Acids Research 43:D588-D592. DOI: 10.1093/nar/gku830.

Camacho C, Coulouris G, Avagyan V, Ma N, Papadopoulos J, Bealer K, Madden TL. 2009. BLAST plus: architecture and applications. BMC Bioinformatics 10:1. DOI: Artn 421 \nDoi 10.1186/1471-2105-10-421.

Chih-Wei Hsu, Chih-Chung Chang and C-JL. 2008. A Practical Guide to Support Vector Classification. BJU international 101:1396-400. DOI: 10.1177/02632760022050997.

Cui G, Fang C, Han K. 2012. Prediction of protein-protein interactions between viruses and human by an SVM model. BMC Bioinformatics 13:11-14. DOI: 10.1186/1471-2105-13-S7S5.

Dee KC, Puleo DA, Bizios R. 2002. An Introduction To Tissue-Biomaterial Interactions. New York, USA: John Wiley \& Sons, Inc. DOI: 10.1002/0471270598.

Donald CL, Brennan B, Cumberworth SL, Rezelj V V., Clark JJ, Cordeiro MT, Freitas de Oliveira França R, Pena LJ, Wilkie GS, Da Silva Filipe A, Davis C, Hughes J, Varjak M, Selinger M, Zuvanov L, Owsianka AM, Patel AH, McLauchlan J, Lindenbach BD, Fall G, Sall AA, Biek R, Rehwinkel J, Schnettler E, Kohl A. 2016. Full Genome Sequence and sfRNA Interferon Antagonist Activity of Zika Virus from Recife, Brazil. PLoS Neglected Tropical Diseases 10. DOI: 10.1371/journal.pntd.0005048.

Dyer MD, Murali TM, Sobral BW. 2007. Computational prediction of host-pathogen proteinprotein interactions. Bioinformatics 23:159-166. DOI: 10.1093/bioinformatics/btm208.

PeerJ reviewing PDF | (2020:11:55456:3:0:NEW 20 Jun 2021) 
643 Dyer MD, Murali TM, Sobral BW. 2011. Supervised learning and prediction of physical 644 interactions between human and HIV proteins. Infection, Genetics and Evolution 11:917-

645

646

647

648

649

650

651

652

653

654

655

656

657

658

659

660

661

662

663

664

665

666

667

668

669

670

671

672

673

674

675

676

677

678

679

680

681

682

683

684

685

686

687

688 923. DOI: 10.1016/j.meegid.2011.02.022.

Eid F-E, ElHefnawi M, Heath LS. 2016. DeNovo: virus-host sequence-based protein-protein interaction prediction. Bioinformatics 32:1144-1150. DOI: 10.1093/bioinformatics/btv737.

Esteves E, Rosa N, Correia MJ, Arrais JP, Barros M. 2017. New Targets for Zika Virus Determined by Human-Viral Interactomic: A Bioinformatics Approach. BioMed Research International 2017. DOI: 10.1155/2017/1734151.

Faye O, Freire CCM, Iamarino A, Faye O, Velasco J, De Oliveira C, Diallo M, Zanotto PMA, Sall AA. 2014. Molecular Evolution of Zika Virus during Its Emergence in the 20 th Century. PLoS Negl Trop Dis 8. DOI: 10.1371/journal.pntd.0002636.

Filippakis H, Spandidos DA, Sourvinos G. 2010. Herpesviruses: Hijacking the Ras signaling pathway. Biochimica et Biophysica Acta - Molecular Cell Research 1803:777-785. DOI: 10.1016/j.bbamcr.2010.03.007.

Fu L, Niu B, Zhu Z, Wu S, Li W. 2012. CD-HIT: Accelerated for clustering the next-generation sequencing data. Bioinformatics 28:3150-3152. DOI: 10.1093/bioinformatics/bts565.

Gajowniczek K, Grzegorczyk I, Ząbkowski T, Bajaj C. 2020. Weighted random forests to improve arrhythmia classification. Electronics (Switzerland) 9:1-20. DOI:

10.3390/electronics9010099.

Ganapathiraju MK, Karunakaran KB, Correa-Menéndez J. 2016. Predicted protein interactions of IFITMs which inhibit Zika virus infection. F1000Research 5:1919. DOI: 10.12688/f1000research.9364.1.

Goodacre N, Devkota P, Bae E, Wuchty S, Uetz P. 2020. Protein-protein interactions of human viruses. Seminars in Cell \& Developmental Biology 99:31-39. DOI:

10.1016/j.semcdb.2018.07.018.

Guedes DR, S Paiva MH, A Donato MM, Barbosa PP, Krokovsky L, dos S Rocha SW, A Saraiva KL, Crespo MM, R Barbosa RM, F Oliveira CM, V Melo-Santos MA, Pena L, Cordeiro MT, de O França RF, Oliveira D, Leal WS, Peixoto CA, J Ayres CF. 2017. Zika virus replication in the mosquito Culex quinquefasciatus in Brazil. Emerging Microbes \& Infections 6. DOI: 10.1101/073197.

Harrington ED, Jensen LJ, Bork P. 2008. Predicting biological networks from genomic data. FEBS letters 582:1251-8. DOI: 10.1016/j.febslet.2008.02.033.

Hazin AN, Poretti A, Martelli CMT, Huisman TA. 2016. Case 41-2015: A Boy with Immune and Liver Abnormalities. New England Journal of Medicine 374:2192-2193. DOI: 10.1056/NEJMc1601156.

Ho J, Moyes DL, Tavassoli M, Naglik JR. 2017. The Role of ErbB Receptors in Infection. Trends in Microbiology 25:942-952. DOI: 10.1016/j.tim.2017.04.009.

Jansen R, Gerstein M. 2004. Analyzing protein function on a genomic scale: the importance of gold-standard positives and negatives for network prediction. Current opinion in microbiology 7:535-45. DOI: 10.1016/j.mib.2004.08.012.

Jiao X, Sherman BT, Huang DW, Stephens R, Baseler MW, Lane HC, Lempicki RA. 2012. DAVID-WS: A stateful web service to facilitate gene/protein list analysis. Bioinformatics 28:1805-1806. DOI: 10.1093/bioinformatics/bts251.

Kanehisa M. 2019. Toward understanding the origin and evolution of cellular organisms. Protein Science:1947-1951. DOI: 10.1002/pro.3715.

Kanehisa M, Sato Y, Furumichi M, Morishima K, Tanabe M. 2019. New approach for

Peer] reviewing PDF | (2020:11:55456:3:0:NEW 20 Jun 2021) 
689

690

691

692

693

694

695

696

697

698

699

700

701

702

703

704

705

706

707

708

709

710

711

712

713

714

715

716

717

718

719

720

721

722

723

724

725

726

727

728

729

730

731

732

733

734

understanding genome variations in KEGG. Nucleic Acids Research 47:D590-D595. DOI: 10.1093/nar/gky962.

Kisseleva T, Bhattacharya S, Braunstein J, Schindler CW. 2002. Signaling through the JAK/STAT pathway, recent advances and future challenges. Gene 285:1-24. DOI: 10.1016/S0378-1119(02)00398-0.

Krakow D. 2013. The dysostoses. In: Emery and Rimoin's Principles and Practice of Medical Genetics. Elsevier, 1-22. DOI: 10.1016/B978-0-12-383834-6.00167-1.

Kuhn M. 2013. Predictive Modeling with R and the caret Package. useR!

Lasso G, Mayer S V, Winkelmann ER, Chu T, Elliot O, Patino-Galindo JA, Park K, Rabadan R, Honig B, Shapira SD. 2019. A Structure-Informed Atlas of Human-Virus Interactions. Cell 178:1526-1541.e16. DOI: 10.1016/j.cell.2019.08.005.

Lawrence DMP, Patterson CE, Gales TL, D'orazio JL, Vaughn MM, Rall GF. 2000. Measles Virus Spread between Neurons Requires Cell Contact but Not CD46 Expression, Syncytium Formation, or Extracellular Virus Production.

Lian X, Yang S, Li H, Fu C, Zhang Z. 2019. Machine-Learning-Based Predictor of HumanBacteria Protein-Protein Interactions by Incorporating Comprehensive Host-Network Properties. Journal of Proteome Research 18:2195-2205. DOI: 10.1021/acs.jproteome.9b00074.

Lima MC, Mendonça L, Rezende AM, Carrera RM, Anibal-Silva CE, Demers M, D'Aiuto L, Wood J, Chowdari K, Griffiths M, Lucena-Araujo AR, Barral-Neto M, Azevedo E, Alves RW, Farias P, Marques ET de A, Castanha P, Donald C, Kohl A, Nimgaonkar V, Franca RFDO. 2019. The transcriptional and protein profile from human infected neuroprogenitor cells is strongly correlated to Zika virus microcephaly cytokines phenotype evidencing a persistent inflammation in the CNS. Frontiers in Immunology 10. DOI: 10.3389/FIMMU.2019.01928.

Limonta D, Jovel J, Kumar A, Airo AM, Hou S, Saito L, Branton W, Wong GKS, Mason A, Power C, Hobman TC. 2018. Human fetal astrocytes infected with zika virus exhibit delayed apoptosis and resistance to interferon: Implications for persistence. Viruses 10. DOI: $10.3390 / \mathrm{v} 10110646$.

Lowe R, Barcellos C, Brasil P, Cruz OG, Honório NA, Kuper H, Carvalho MS. 2018. The zika virus epidemic in brazil: From discovery to future implications. International Journal of Environmental Research and Public Health 15. DOI: 10.3390/ijerph15010096.

Mei S, Zhang K. 2019. Neglog: Homology-based negative data sampling method for genomescale reconstruction of human protein-protein interaction networks. International Journal of Molecular Sciences 20. DOI: 10.3390/ijms20205075.

Mothes W, Sherer NM, Jin J, Zhong P. 2010. Virus Cell-to-Cell Transmission. Journal of Virology 84:8360-8368. DOI: 10.1128/jvi.00443-10.

Murray KO, Gorchakov R, Carlson AR, Berry R, Lai L, Natrajan M, Garcia MN, Correa A, Patel SM, Aagaard K, Mulligan MJ. 2017. Prolonged detection of zika virus in vaginal secretions and whole blood. Emerging Infectious Diseases 23:99-101. DOI: 10.3201/eid2301.161394.

Nambala P, Su W-C. 2018. Role of Zika Virus prM Protein in Viral Pathogenicity and Use in Vaccine Development. Frontiers in Microbiology 9:1-6. DOI: 10.3389/fmicb.2018.01797.

Nan Y, Wu C, Zhang YJ. 2017. Interplay between Janus kinase/signal transducer and activator of transcription signaling activated by type I interferons and viral antagonism. Frontiers in Immunology 8:1-17. DOI: 10.3389/fimmu.2017.01758.

Ogata H, Goto S, Sato K, Fujibuchi W, Bono H, Kanehisa M. 1999. KEGG: Kyoto encyclopedia

Peer) reviewing PDF | (2020:11:55456:3:0:NEW 20 Jun 2021) 
735

736

737

738

739

740

741

742

743

744

745

746

747

748

749

750

751

752

753

754

755

756

757

758

759

760

761

762

763

764

765

766

767

768

769

770

771

772

773

774

775

776

777

778

779

780

of genes and genomes. Nucleic Acids Research 27:29-34. DOI: 10.1093/nar/27.1.29.

Pavithra L. Chavali 1, *† Lovorka Stojic, $1 *$ Luke W. Meredith, 2 Nimesh Joseph 1, Michael S. Nahorski 3, J. T, Sanford 2, Trevor R. Sweeney 2, Ben A. Krishna, 4 Myra Hosmillo 2, Andrew E. Firth 2, Richard Bayliss 5, L. C, Marcelis 6, Susan Lindsay 7, Ian Goodfellow, 2 C. Geoffrey Woods 3, Gergely F. 2017. Neurodevelopmental protein Musashi 1 interacts with the Zika genome and promotes viral replication. Science (New York, N.Y.).

Pickett BE, Sadat EL, Zhang Y, Noronha JM, Squires RB, Hunt V, Liu M, Kumar S, Zaremba S, Gu Z, Zhou L, Larson CN, Dietrich J, Klem EB, Scheuermann RH. 2012. ViPR: An open bioinformatics database and analysis resource for virology research. Nucleic Acids Research 40:593-598. DOI: 10.1093/nar/gkr859.

Rawlings JS, Rosler KM, Harrison DA. 2004. The JAK/STAT signaling pathway. Journal of Cell Science 117:1281-1283. DOI: 10.1242/jcs.00963.

Rigau-Pérez JG, Clark GG, Gubler DJ, Reiter P, Sanders EJ, Vance Vorndam A. 1998. Dengue and dengue haemorrhagic fever. The Lancet 352:971-977. DOI: 10.1016/S01406736(97)12483-7.

Saiz J-C, Vázquez-Calvo Á, Blázquez AB, Merino-Ramos T, Escribano-Romero E, MartínAcebes MA. 2016. Zika virus: The latest newcomer. Frontiers in Microbiology 7. DOI: 10.3389/fmicb.2016.00496.

Scaturro P, Stukalov A, Haas DA, Cortese M, Draganova K, Płaszczyca A, Bartenschlager R, Götz M, Pichlmair A. 2018. An orthogonal proteomic survey uncovers novel Zika virus host factors. Nature 561:253-257. DOI: 10.1038/s41586-018-0484-5.

Scher MS. 2019. Fetal neurology: Principles and practice with a life-course perspective. In: Handbook of Clinical Neurology. Elsevier B.V., 1-29. DOI: 10.1016/B978-0-444-640291.00001-1.

Šsestan M, Marinović S, Kavazović I, Cekinović Đ, Wueest S, Turk Wensveen T, Brizić I, Jonjić S, Konrad D, Wensveen FM, Polić B. 2018. Virus-Induced Interferon- $\gamma$ Causes Insulin Resistance in Skeletal Muscle and Derails Glycemic Control in Obesity. Immunity 49:164177.e6. DOI: 10.1016/j.immuni.2018.05.005.

Shah PS, Link N, Jang GM, Sharp PP, Zhu T, Swaney DL, Johnson JR, Von Dollen J, Ramage HR, Satkamp L, Newton B, Hüttenhain R, Petit MJ, Baum T, Everitt A, Laufman O, Tassetto M, Shales M, Stevenson E, Iglesias GN, Shokat L, Tripathi S, Balasubramaniam V, Webb LG, Aguirre S, Willsey AJ, Garcia-Sastre A, Pollard KS, Cherry S, Gamarnik A V., Marazzi I, Taunton J, Fernandez-Sesma A, Bellen HJ, Andino R, Krogan NJ. 2018. Comparative Flavivirus-Host Protein Interaction Mapping Reveals Mechanisms of Dengue and Zika Virus Pathogenesis. Cell 175:1931-1945.e18. DOI: 10.1016/j.cell.2018.11.028.

Shannon P, Markiel A, Ozier O, Baliga NS, Wang JT, Ramage D, Amin N, Schwikowski B, Ideker T. 2003. Cytoscape: A software Environment for integrated models of biomolecular interaction networks. Genome Research 13:2498-2504. DOI: 10.1101/gr.1239303.

Shen J, Zhang J, Luo X, Zhu W, Yu K, Chen K, Li Y, Jiang H. 2007. Predicting protein-protein interactions based only on sequences information. Proceedings of the National Academy of Sciences of the United States of America 104:4337-41. DOI: 10.1073/pnas.0607879104.

Sher AA, Glover KKM, Coombs KM. 2019. Zika virus infection disrupts astrocytic proteins involved in synapse control and axon guidance. Frontiers in Microbiology 10. DOI: 10.3389/fmicb.2019.00596.

Shoji I. 2012. Roles of the two distinct proteasome pathways in hepatitis C virus infection. World Journal of Virology 1:44. DOI: 10.5501/wjv.v1.i2.44. 
781

782

783

784

785

786

787

788

789

790

791

792

793

794

795

796

797

798

799

800

801

802

803

804

805

806

807

808

809

810

811

812

813

814

815

816

817

818

819

820

821

822

823

824

825

826

Singhal M, Resat H. 2007. A domain-based approach to predict protein-protein interactions. BMC Bioinformatics 8. DOI: 10.1186/1471-2105-8-199.

Sirohi D, Chen Z, Sun L, Klose T, Pierson TC, Rossmann MG, Kuhn RJ. 2016. The 3.8 A resolution cryo-EM structure of Zika virus. Science 352:467-470. DOI: 10.1126/science.aaf5316.

Souza WV de, Araújo TVB de, Albuquerque M de FPM, Braga MC, Ximenes RA de A, Miranda-Filho D de B, Bezerra LCA, Dimech GS, Carvalho PI de, Assunção RS de, Santos RH, Oliveira WK de, Rodrigues LC, Martelli CMT. 2016. Microcephaly in Pernambuco State, Brazil: epidemiological characteristics and evaluation of the diagnostic accuracy of cutoff points for reporting suspected cases. Cadernos de saude publica 32:e00017216. DOI: 10.1590/0102-311X00017216.

Spitz D. 2019. Zika: the continuing threat. Bulletin of the World Health Organization 97:6-7. DOI: $10.2471 /$ BLT.19.020119.

Statnikov A, Aliferis CF. 2007. Are random forests better than support vector machines for microarray-based cancer classification? AMIA ... Annual Symposium proceedings / AMIA Symposium. AMIA Symposium:686-690.

Statnikov A, Wang L, Aliferis CF. 2008. A comprehensive comparison of random forests and support vector machines for microarray-based cancer classification. BMC Bioinformatics 9:1-10. DOI: 10.1186/1471-2105-9-319.

Villaveces JM, Jiménez RC, Porras P, Del-Toro N, Duesbury M, Dumousseau M, Orchard S, Choi H, Ping P, Zong NC, Askenazi M, Habermann BH, Hermjakob H. 2015. Merging and scoring molecular interactions utilising existing community standards: Tools, use-cases and a case study. Database 2015. DOI: 10.1093/database/bau131.

WHO. 2016.WHO statement on the first meeting of the International Health Regulations (2005) (IHR 2005) Emergency Committee on Zika virus and observed increase in neurological disorders and neonatal malformations. Available at http://www.who.int/en/newsroom/detail/01-02-2016-who-statement-on-the-first-meeting-of-the-international-healthregulations-(2005)-(ihr-2005)-emergency-committee-on-zika-virus-and-observed-increasein-neurological-disorders-and-neonatal-malformations

Widman DG, Young E, Yount BL, Plante KS, Gallichotte EN, Carbaugh DL, Peck KM, Plante J, Swanstrom J, Heise MT, Lazear HM, Baric RS. 2017. A reverse genetics platform that spans the Zika virus family tree. mBio 8. DOI: 10.1128/mBio.02014-16.

Xiao N, Cao D, Min-Feng Z, Xu Q. 2015. protr : R package for generating various numerical representation schemes of protein sequence. Bioinformatics 31:1857-1859.

Yang S, Li H, He H, Zhou Y, Zhang Z. 2019. Critical assessment and performance improvement of plant-pathogen protein-protein interaction prediction methods. Briefings in Bioinformatics 20:1-14. DOI: 10.1093/bib/bbx123.

Yang X, Yang S, Li Q, Wuchty S, Zhang Z. 2020. Prediction of human-virus protein-protein interactions through a sequence embedding-based machine learning method. Computational and Structural Biotechnology Journal 18:153-161. DOI: 10.1016/j.csbj.2019.12.005.

Yoon KJ, Song G, Qian X, Pan J, Xu D, Rho HS, Kim NS, Habela C, Zheng L, Jacob F, Zhang F, Lee EM, Huang WK, Ringeling FR, Vissers C, Li C, Yuan L, Kang K, Kim S, Yeo J, Cheng Y, Liu S, Wen Z, Qin CF, Wu Q, Christian KM, Tang H, Jin P, Xu Z, Qian J, Zhu H, Song H, Ming G li. 2017. Zika-Virus-Encoded NS2A Disrupts Mammalian Cortical Neurogenesis by Degrading Adherens Junction Proteins. Cell Stem Cell 21:349-358.e6. DOI: $10.1016 /$ j.stem.2017.07.014.

PeerJ reviewing PDF | (2020:11:55456:3:0:NEW 20 Jun 2021) 
827 Yu I-M, Holdaway HA, Chipman PR, Kuhn RJ, Rossmann MG, Chen J. 2009. Association of 828 the pr Peptides with Dengue Virus at Acidic pH Blocks Membrane Fusion. Journal of 829 Virology 83:12101-12107. DOI: 10.1128/jvi.01637-09.

830 Zheng A, Umashankar M, Kielian M. 2010. In Vitro and In vivo studies identify important 831 features of dengue virus pr-E protein interactions. PLoS Pathogens 6. DOI:

$832 \quad 10.1371 /$ journal.ppat.1001157.

833 


\section{Figure 1}

Four steps approach for negative pair of proteins.

The four steps to set a pair of one viral protein " $\mathrm{V}$ " and one host's protein " $\mathrm{H}$ " as proteins that does not interact. First step (A) verifies if the pair of proteins is not listed as a pair on the positive interaction's dataset. Second step (B) consists in verify if the " $\mathrm{V}$ " protein does not interact with another host's protein " $\mathrm{Hx}$ " that has more than $20 \%$ identity to "H". Third step (C) checks also if the protein " $\mathrm{H}$ " does not interact with another viral protein " $\mathrm{Vx}$ " that has more than $20 \%$ identity to "V". Fourth step (D) verifies if another pair, composed by a viral protein " $\mathrm{A}$ " that has at least $40 \%$ of identity with " $\mathrm{V}$ " and a host protein "B" that has at least $40 \%$ of identity with " $\mathrm{H}$ ", is listed as positive interaction in the positive dataset. The pair " $\mathrm{V}$ $\mathrm{H}$ " is discarded from negative dataset if it has at least $40 \%$ identity with pair "A-B" and "A-B" is listed in the positive dataset. 

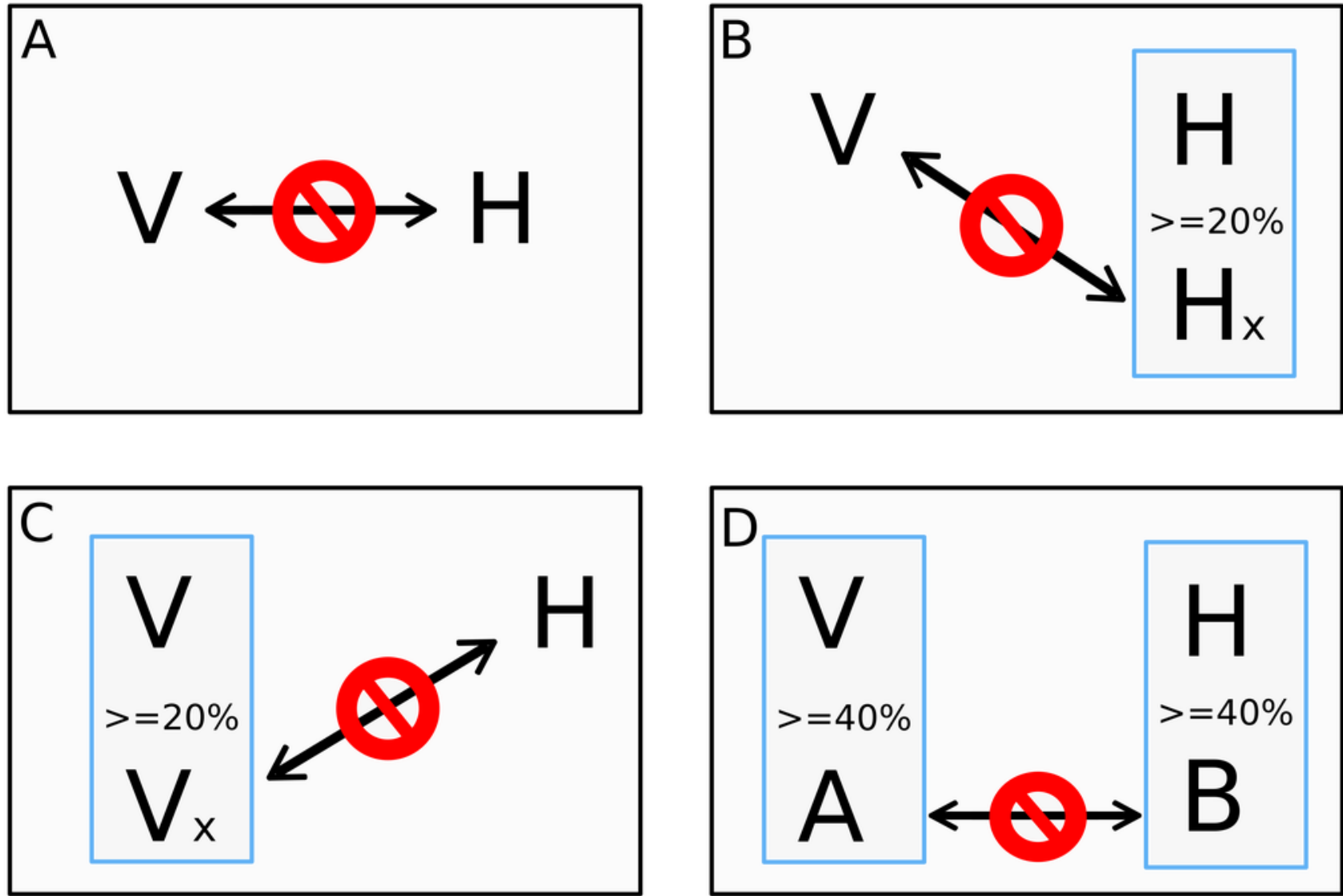
Figure 2

Number of interactions predicted.

The number of interactions predicted by the consensus prediction was analyzed and was described, distributing the interactions by strain. Red and blue rectangles are structural and nonstructural ZIKV proteins, respectively.

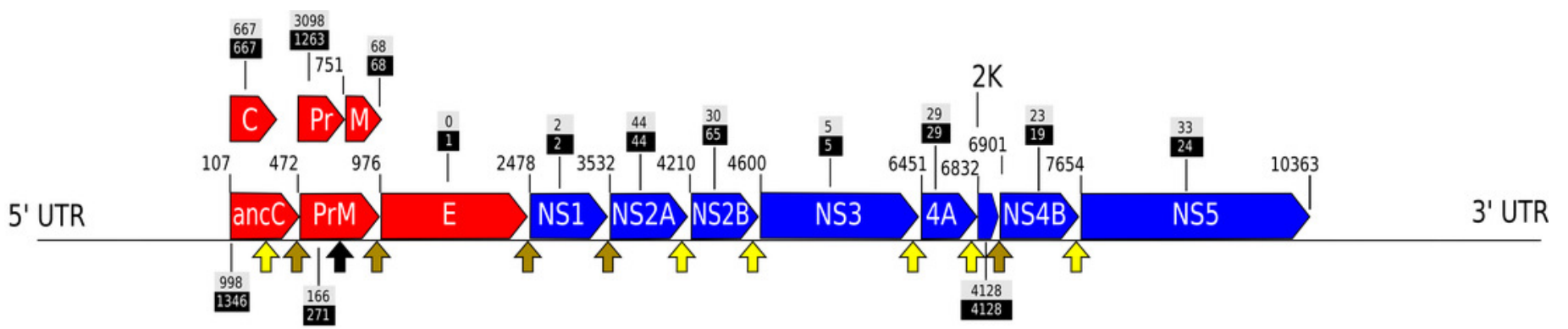

\# PE243 interactions

\section{\#FSS13025 interactions}

$\Rightarrow$ Host protease

$\Rightarrow$ Furin

$\Rightarrow$ Viral protease 
Figure 3

Numbers of interactions per strain.

The Venn Diagram shows the interactions of human proteins per ZIKV strain. The model predicted 25 exclusive interactions for strain FSS13025 while 1,384 exclusive interactions were predicted for strain PE243. The model predicted yet 15,814 interactions for both strains.

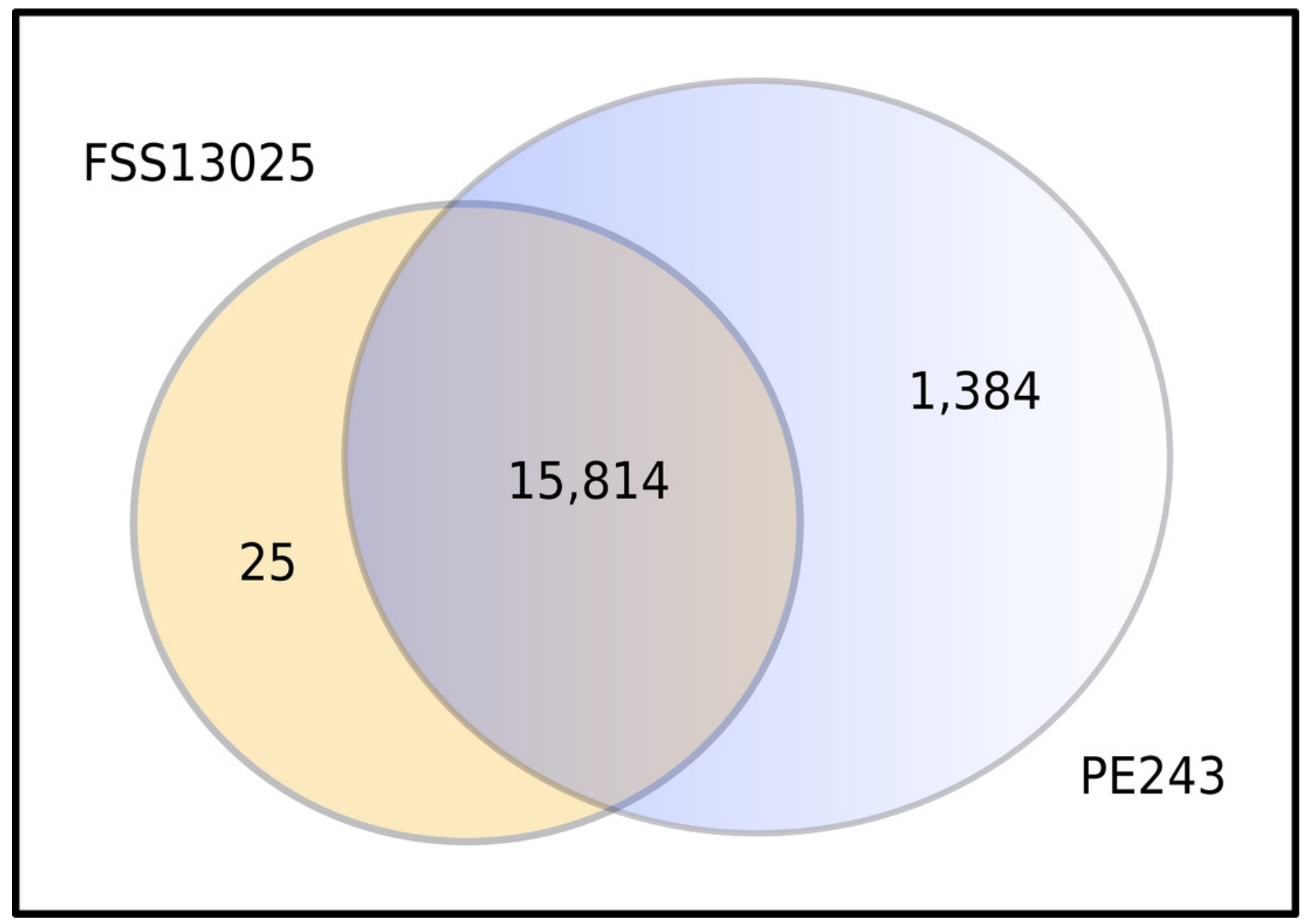


Figure 4

Enriched pathways with statistical relevance.

Figure 4 presents a dot plot graphic for each strain. The size of each dot represents the number of genes ("Count") from the consensus prediction enriched in each pathway and the color presents the statistical relevance based on the FDR.
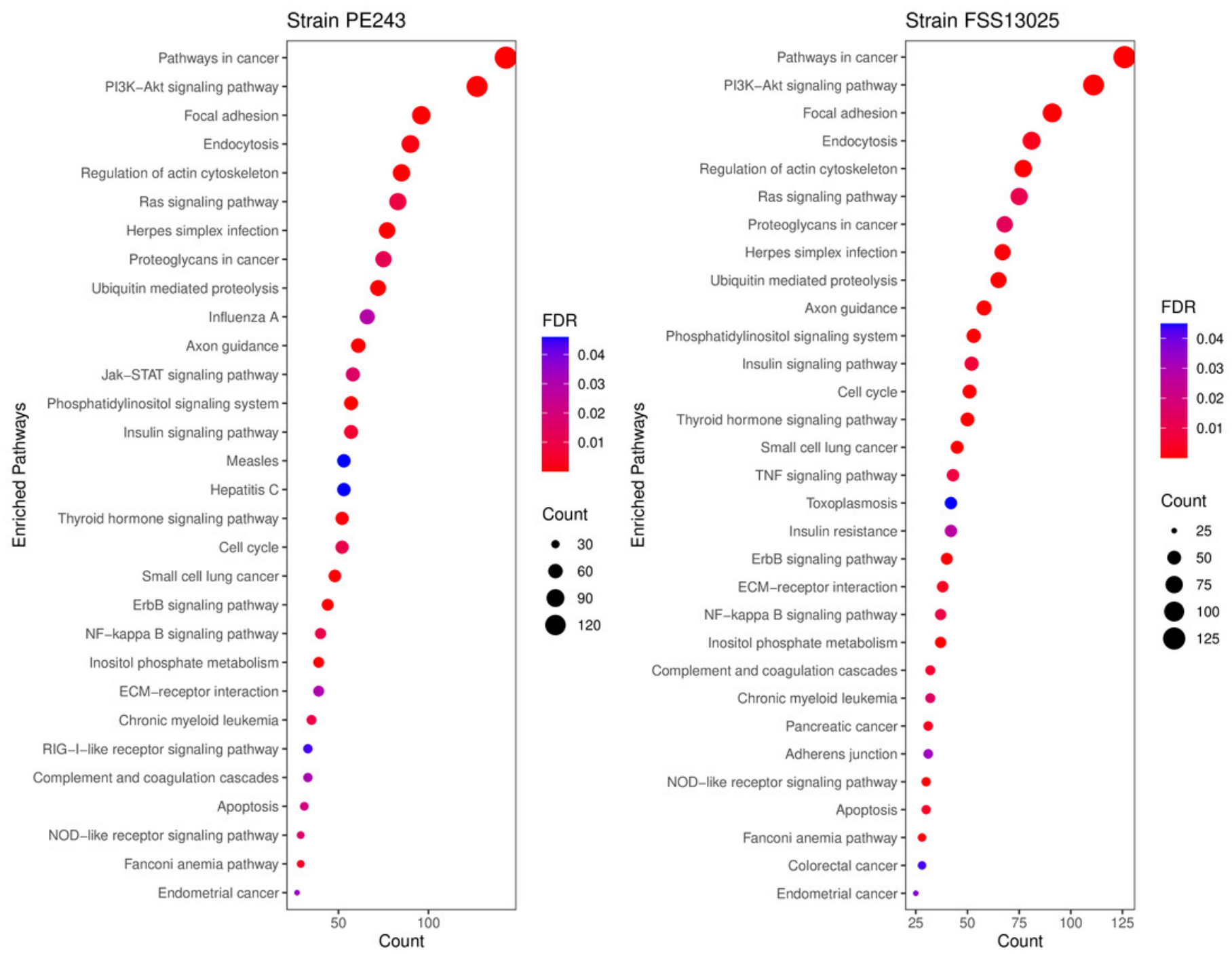
Figure 5

ErbB signaling pathway map.

Figure 5 shows ErbB signaling pathway map. Enriched proteins in our data were marked in green over the pathway map. Green boxes represent proteins that were enriched by DAVID analysis between ZIKV (PE243 strain) and $H$. sapiens interactions.

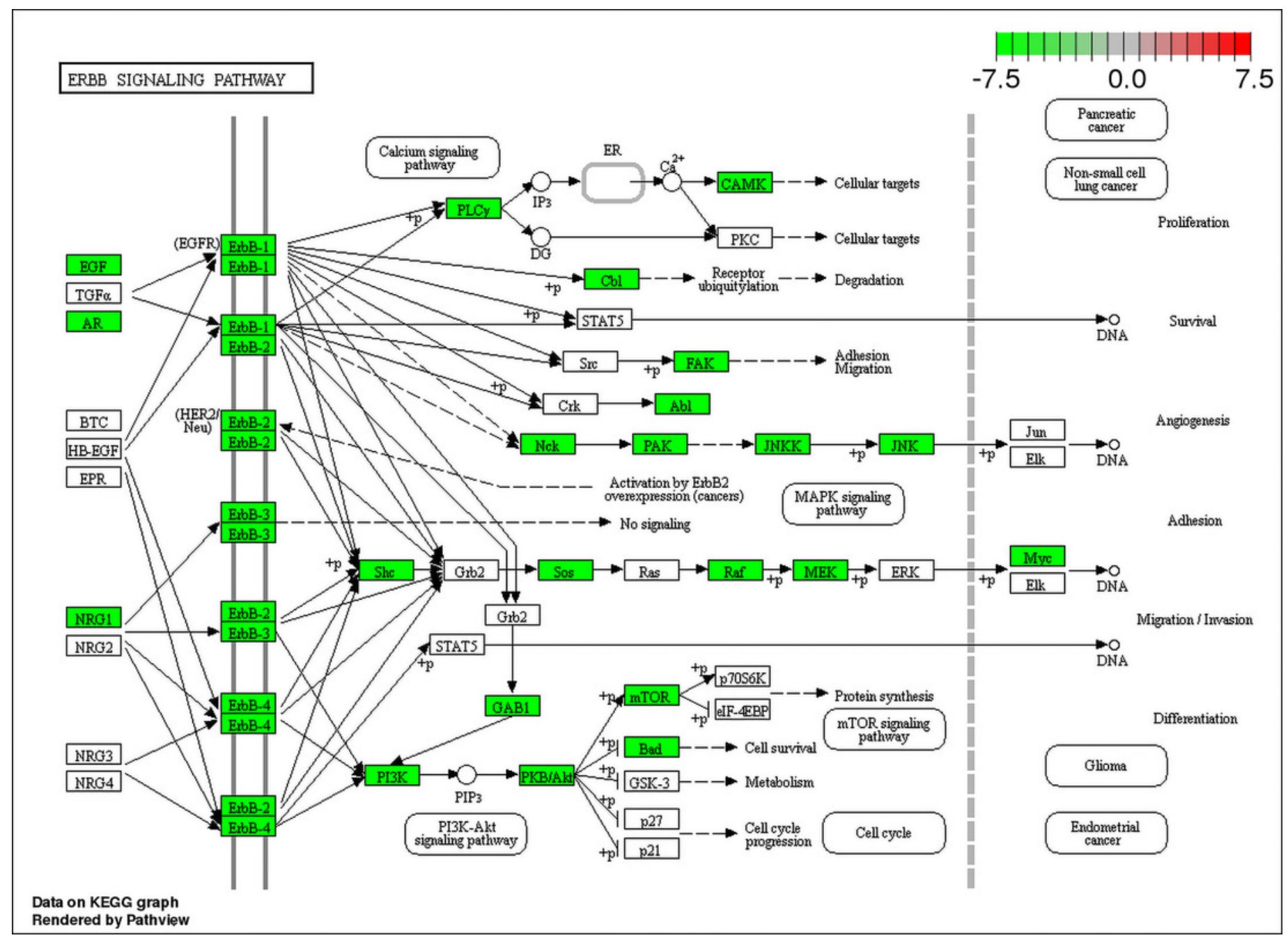




\section{Table 1 (on next page)}

Classification of amino acids in group labels. 
Table 1. Classification of amino acids in group labels.

\begin{tabular}{cc}
\hline Amino acid & Group Label \\
\hline$\{A, V, G\}$ & 1 \\
$\{I, L, F, P\}$ & 2 \\
$\{Y, M, T, S\}$ & 3 \\
$\{H, N, Q, W\}$ & 4 \\
$\{R, K\}$ & 5 \\
$\{D, E\}$ & 6 \\
$\{C\}$ & 7 \\
\hline
\end{tabular}

2 